\title{
Formes vivantes
}

\section{Living forms}

\author{
Jean-Charles Hameau', Kimberley Harthoorn ${ }^{2}$ \\ ${ }^{1}$ Conservateur du patrimoine, Musée national Adrien Dubouché, jean-charles.hameau@sevresciteceramique.fr \\ ${ }^{2}$ Historienne de l'art, kimberley.harthoorn@limogesciteceramique.fr
}

RÉSUMÉ. L'exposition Formes vivantes, qui ouvrira le 9 octobre 2019 au Musée national Adrien Dubouché à Limoges, se donne pour objectif d'explorer les relations entre céramique et vivant, de la Renaissance à nos jours.

Le présent article vise à exposer les axes principaux de ce projet d'exposition, mêlant histoire des arts décoratifs et histoire des sciences : la production naturaliste, de l'image ressemblante au moulage sur le vif ; les œuvres qui relèvent d'un imaginaire organique recomposé à partir des formes de la nature ; l'influence des révolutions visuelles que sont la mise au jour du vivant invisible, dans ses dimensions anatomique, microscopique ou génétique.

On voit alors que la céramique est un domaine innovant de la création contemporaine, mais aussi que le matériau céramique lui-même, par ses propriétés physiques, est apte à jouer un rôle dans les passages de frontière entre vivant et non-vivant, tant conceptuellement que concrètement, notamment avec l'essor de la céramique biomédicale.

Une telle démarche transdisciplinaire, inscrite dans l'actualité de la création et de la recherche, nous permet ainsi d'explorer l'évolution de notre vision du vivant, à la lumière des dernières découvertes scientifiques, mais aussi du point de vue politique, éthique, et esthétique.

ABSTRACT. The Formes vivantes (Living forms) exhibition, which will open on October 9th, 2019 at the Musée national Adrien Dubouché, Limoges, France, aims at exploring the relationship between ceramics and the living, from the Renaissance until today.

This article will lay out the main axes of the exhibition project : naturalist objects, from images resembling the living to castings of living beings ; works showing how an organic imagination is born from the recomposition of shapes found in nature ; the influence of visual revolutions that brought to sight invisible dimensions of the living, anatomical, microscopic or genetic.

The exhibition will show that ceramics can also be an innovative realm of contemporary creation. We also hope to demonstrate how the ceramic material in itself, because of its physical and chemical properties, can play a role in crossing boundaries between the living and the non-living, conceptually, but also in practice, for instance with the emergence of biomedical ceramics.

Such a transdisciplinary approach, following the latest developments in artistic creation and scientific research, will allow us to study our relationship to the idea of living, in the light of recent scientific discoveries, but also from a political, ethical and aesthetical point of view.

MOTS-CLÉS. céramique, vivant, naturalisme, morphogenèse, impression 3D, arts décoratifs, transdisciplinarité, éthique, esthétique.

KEYWORDS. ceramics, living, naturalism, morphogenesis, 3D printing, decorative arts, transdisciplinarity, ethics, aesthetics.

\section{Introduction}

\section{Céramique et biologie}

L'idée d'une relation entre la céramique et le vivant fait écho à une hypothèse scientifique sur le rôle joué par l'argile dans les origines de la vie. L'argile a pu être un vecteur du passage du non-vivant au vivant, peut-être en jouant le rôle d'échafaudage ou de réacteur favorisant l'assemblage d'acides aminés .

L'étude des formes vivantes en céramique fait surgir des analogies entre la matière inerte et la matière organique, ainsi que des rapprochements entre des concepts issus de la biologie et le travail des céramistes. 
Selon le potier Jean Girel, «l'homme d'aujourd'hui a envie d'en savoir plus sur sa planète, de comprendre ce qui se passe à l'intérieur, ou autour, sur d'autres, d'explorer l'infiniment grand, l'infiniment petit. La céramique, qui met en forme les transformations de la matière, accomplit précisément ce travail : à l'échelle corpusculaire dans le comportement des argiles, à l'échelle sidérale dans les processus de fusion des émaux ${ }^{2} \gg$. La céramique permet donc de penser au-delà de l'échelle humaine, de se rapprocher de phénomènes incommensurables. Elle invite à penser la matière et ses métamorphoses qui se produisent sur des temps bien plus longs que ceux de l'histoire humaine.

Penser le vivant dans sa globalité implique également d'envisager la matière non comme inerte, mais comme changeante, en constante évolution. On observe alors des ressemblances troublantes entre matières minérales et organismes vivants, à tel point que les catégories de vivant et de non-vivant s'en trouvent sinon menacées, du moins mises en question.

Ce qui justifie l'analogie entre céramique et vivant se situe également dans une commune disposition à devenir forme, mis en forme par l'environnement. Le terme argile définit une famille de minéraux née de l'altération de roches (généralement des silicates), qui tire sa plasticité de sa composition en feuillets qui glissent les uns sur les autres. Par conséquent, la céramique, du fait de cette plasticité, est un terrain privilégié de l'étude de la naissance et du devenir des formes. Le façonnage (modelage, tournage, coulage, impression 3D...) implique une attention aux propriétés physiques de la matière (qualité de l'argile, concentration en eau, «mémoire » de la terre ${ }^{3}$ ). Chez les artistes et les artisans, il devient un moyen d'acquérir des connaissances empiriques sur l'interaction entre matière et forme, une préoccupation qui concerne également les scientifiques par le biais des études sur la morphogenèse.

La céramique impose un effort d'anticipation des transformations irrémédiables qui s'accomplissent lors de la cuisson (retrait, changements de couleur, vitrification). Mais cet art est également nourri par la conscience de l'aléa, des paramètres non maîtrisables du feu qui mettent en doute l'idée d'un contrôle absolu de l'artiste sur sa production. Une tension similaire entre prédictibilité et part aléatoire intervient dans la formation du vivant. Le gène est en effet perçu comme un code ou un texte dans lequel l'organisme à venir serait déjà contenu ${ }^{4}$. La science contemporaine a néanmoins mis en balance les mécanismes du code génétique avec ceux de l'individuation épigénétique. D'autre part, la notion d'écologie ${ }^{5}$ renforce l'idée selon laquelle le vivant doit être envisagé en relation avec son milieu.

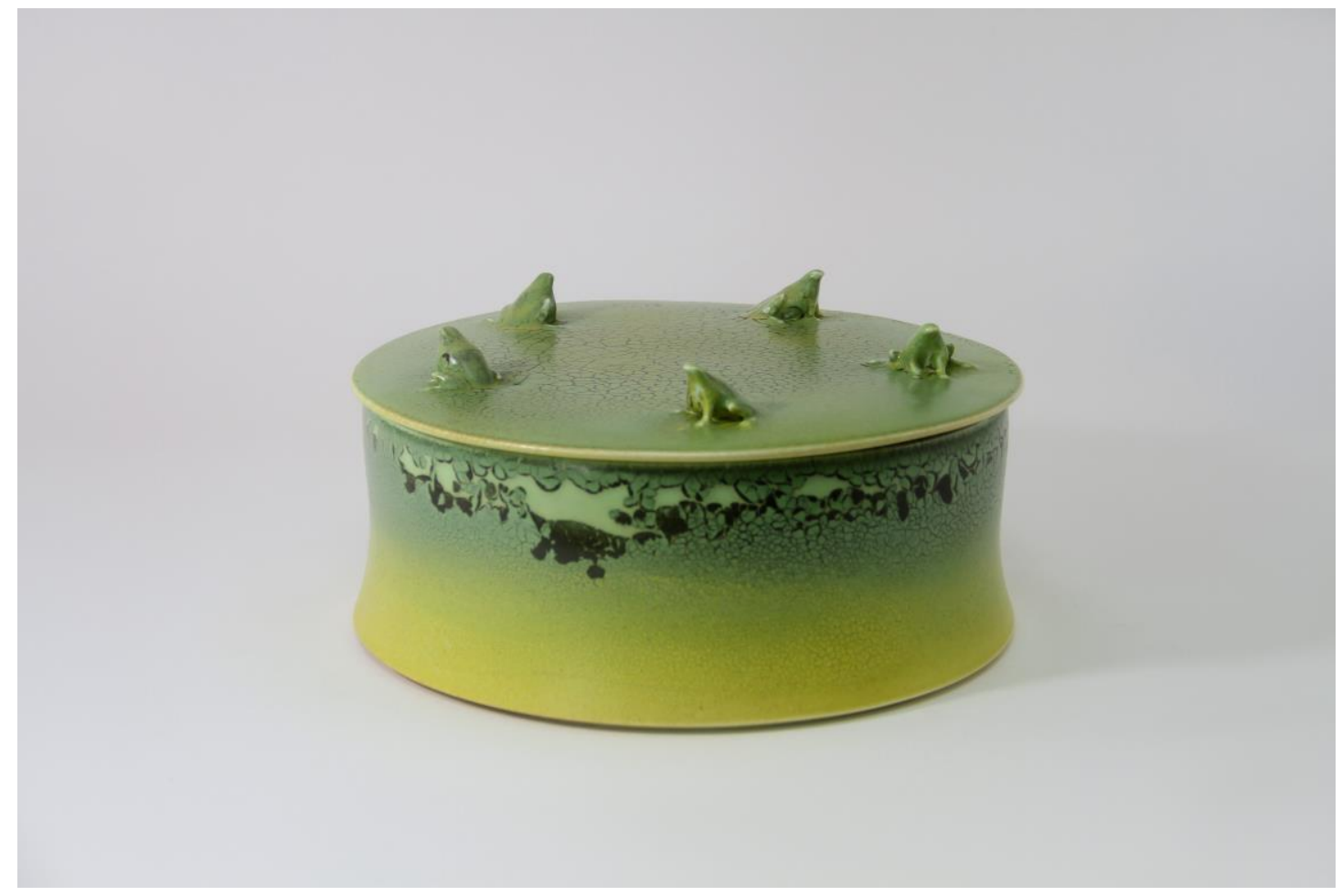

Jean Girel, Pot à décor de peau animale, série « Batraciens », porcelaine, 2012, collection de l'artiste, crédit photo : Jean Girel. 


\section{L'idée d'une exposition}

De nombreux créateurs d'aujourd'hui s'intéressent à la manière dont le vivant prend forme dans la nature, à l'image de la designer Neri Oxman ou de l'architecte Achim Menges, qui s'en emparent pour inventer des outils, des méthodes et des œuvres ${ }^{6}$. La scène céramique contemporaine internationale n'échappe pas à cette confrontation avec le monde du vivant. Les concrétions florales de Johan Creten, les grotesques agglutinés de Michel Gouéry, les bestiaires émaillés de Jean Girel ou encore les microorganismes d'Arnold Annen témoignent de l'actualité et de la présence proliférante des formes «bioinspirées » dans la création céramique.

L'intérêt pour cette question, loin d'être une nouveauté, s'inscrit dans une longue histoire de la représentation du vivant: grouillant au $\mathrm{XVI}^{\mathrm{e}}$ siècle chez Bernard Palissy, stylisé dans les formes rocaille du XVIIIe siècle ou tendant vers l'abstraction dans l'Art nouveau. Ces échos historiques révèlent une diversité des approches mais aussi certaines constantes liées au medium.

Le Musée national Adrien Dubouché a choisi d'en faire le thème d'une exposition intitulée Formes vivantes qui ouvrira ses portes le 9 octobre 2019, jusqu'au 10 février 2020. S'appuyant sur un corpus d'œuvres occidentales d'une période allant du XVI ${ }^{\mathrm{e}}$ siècle à l'art contemporain, ce projet a moins pour objectif de dresser une chronologie linéaire et exhaustive du sujet que de faire surgir des moments de l'histoire de l'art où se manifeste, en céramique, un regard porté sur le vivant. Il s'agit également de souligner des correspondances formelles et conceptuelles non seulement entre des œuvres d'époques différentes, mais aussi entre des champs disciplinaires souvent cloisonnés. Centrée autour de la céramique, dont le lien spécifique avec le monde organique sera interrogé, cette exposition offrira ainsi des comparaisons avec d'autres techniques et cherchera à nouer un dialogue entre art et science.

Le vivant n'est pas seulement une notion scientifique : c'est aussi un enjeu social et existentiel. L'évolution de la compréhension scientifique du vivant se fait dans le même temps qu'un changement profond de notre rapport au vivant. Un nouvel imaginaire du vivant se construit, généré par la porosité que l'on découvre entre des catégories autrefois isolées les unes des autres, entre vivant et non-vivant, entre animal, végétal et minéral, entre disciplines universitaires. L'un des enjeux de l'exposition Formes vivantes est de montrer que la céramique se situe dans cette zone d'indistinction entre vivant et non-vivant, et que l'y placer permet de générer des questionnements originaux et féconds. Cette approche s'inscrit dans un contexte politique dans lequel le vivant a une importance croissante.

L'exposition s'articulera autour de trois séquences thématiques correspondant à deux approches d'abord étudiées séparément puis conjointement, lors d'un zoom sur les processus cachés du vivant.

Dans un premier temps, la notion de «naturalisme» sera abordée au regard de techniques céramiques qui impliquent l'observation voire la captation des formes du vivant. Loin d'être univoque, le rapport à la nature qui se manifeste dans les pièces étudiées s'inscrit toujours dans un contexte historique et scientifique précis.

Au-delà du défi consistant à imiter la nature, l'observation des processus du vivant donne naissance à un imaginaire organique proliférant qui colonise l'art, et en particulier la céramique, assez plastique pour se prêter aisément au jeu des formes.

L'exposition s'attachera enfin à montrer comment cette tension entre naturalisme et imaginaire s'introduit à l'intérieur du vivant via la céramique, à la faveur de connaissances scientifiques (anatomie, génétique) et de technologies (microscopie, informatique, impression 3D) qui rendent visible l'intérieur des corps, ou les corps invisibles à l'œil nu. 


\section{Naturalisme et céramique}

En tant que méthode artistique, le naturalisme désigne l'imitation exacte de la nature et s'oppose à la stylisation ou au symbolisme. Loin d'être réductibles à de simples copies, les céramiques naturalistes traduisent un désir de se mesurer à l'infinie complexité des formes de la faune et de la flore. Célébration de la beauté du vivant, défi technique ou expression d'une pensée scientifique, ces pièces rendent compte d'un regard sur la nature et d'une vision du monde.

Les « rustiques figulines » et les décors de grotte du célèbre potier Bernard Palissy (1510-1590) s'éclairent lorsqu'on les rapproche de ses observations de la nature et des phénomènes qui la régissent. La technique du moulage sur le vif inscrit sa démarche artistique dans le contexte de la redécouverte et de l'étude des formes de la nature à la Renaissance. L'histoire naturelle se constitue en tant que champ du savoir dès les années $1530^{7}$. Les universités établissent des chaires dédiées, à Padoue, notamment. Elles organisent des cours, comme ceux du médecin et professeur Guillaume Rondelet (1507-1566) à Montpellier, qui publie en 1554 le De piscibus marinis ${ }^{8}$, un ouvrage illustré sur les poissons, connu de Palissy9. Chez ce dernier, les expériences qu'il mène dans le champ de la céramique sont indissociables de sa pensée de la nature dans laquelle terre et minéraux naissent et croissent dans un mouvement qu'il compare au vivant grâce à l'action des « eaux germinatives ${ }^{10}$ ». Mais ses scènes inspirées de la nature sont aussi des trompe-l'œil, des jeux rhétoriques sur l'illusion et la réalité chargés de références religieuses et littéraires typiques de l'Europe maniériste.

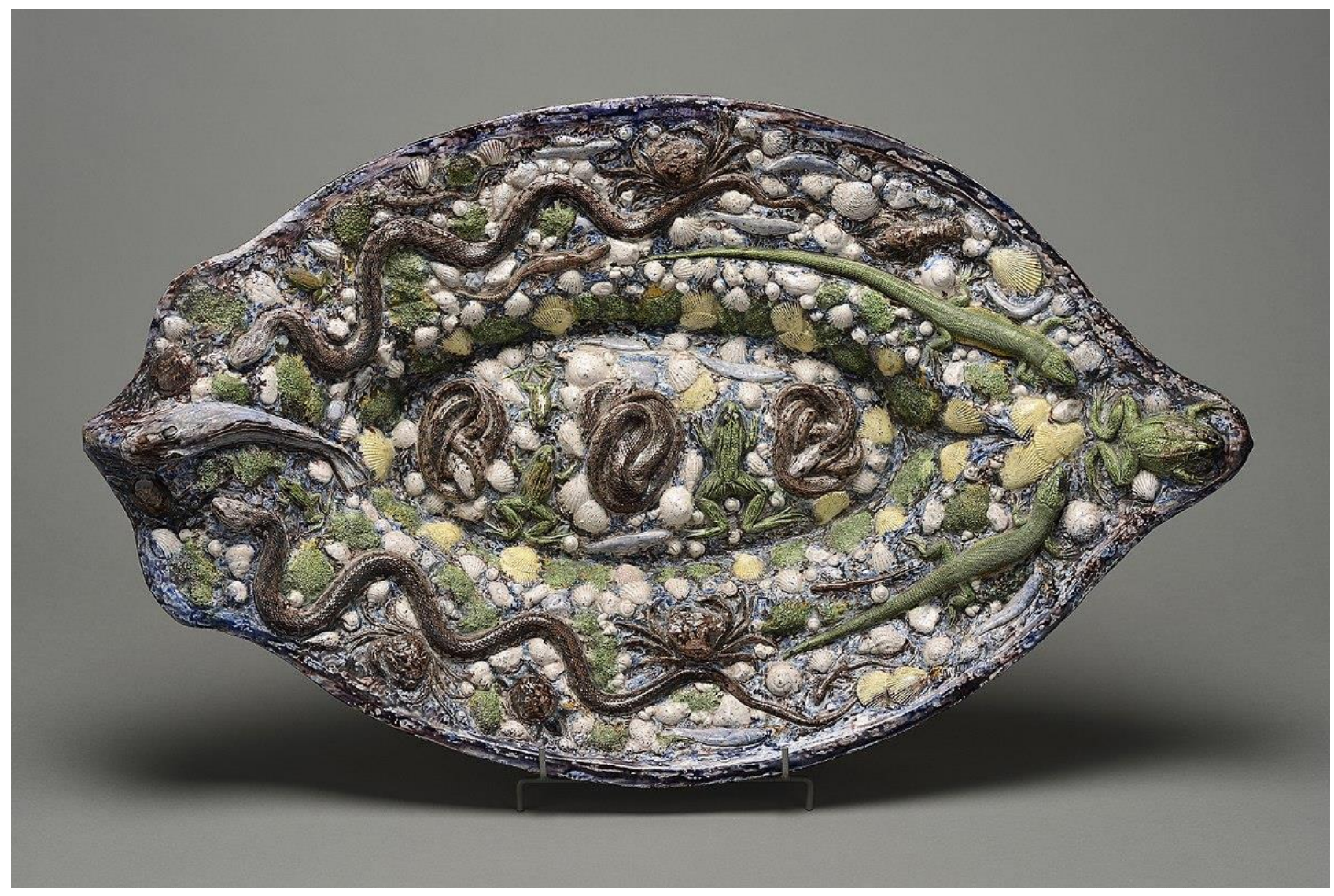

Bernard Palissy, Plat à décor de rustiques figulines, terre vernissée, seconde moitié du XVI siècle, Musée des Beaux-Arts de Lyon, crédit photo : Alain Basset.

Au XIX ${ }^{\mathrm{e}}$ siècle, des artistes tels que Charles-Jean Avisseau (1796-1861), Georges Pull (1810-1889) ou Victor Barbizet (1805-1870) remettent à la mode le langage artistique de Bernard Palissy, dans le contexte de l'historicisme, plus précisément du style néo-Renaissance, qui touche les arts décoratifs à cette époque. Reprenant plus ou moins fidèlement la technique de Bernard Palissy, ils s'attellent à égaler, voire à dépasser ses œuvres, notamment par la taille, la richesse des couleurs ou la complexité des compositions. Pour Charles-Jean Avisseau, qui entretient chez lui un vivarium et élève des 
batraciens dans une mare, l'observation et la connaissance du vivant demeurent le socle de son travail, mais la mise en scène et la recherche d'effets sculpturaux priment sur la rigueur scientifique dans la représentation des espèces et de leur milieu ${ }^{11}$. Dans ses œuvres, la nature en perpétuelle transformation que célébrait Palissy fait place à un milieu foisonnant, dangereux et sauvage, théâtre de luttes sanglantes, qui revêtent une signification morale liée à la symbolique chrétienne, en particulier le combat entre le Bien et le Mal. Au regard de l'histoire des sciences naturelles du milieu du XIX ${ }^{\mathrm{e}}$ siècle, on peut légitimement se demander si ces œuvres d'art n'anticipent pas la pensée darwinienne de la lutte des espèces pour leur survie ${ }^{12}$.

Outre la représentation figurée de compositions envahies par le vivant, le travail de l'émail est le lieu d'une quête naturaliste qui inspire encore de nombreux potiers contemporains fascinés par la tradition chinoise et l'héritage de Bernard Palissy. L'obtention de nouvelles couleurs et textures nécessite en céramique une recherche lente et précise, et bien souvent, des connaissances théoriques et pratiques en chimie. La céramique s'approche en cela des sciences naturelles dont elle s'approprie bien souvent la méthode et l'objet d'étude. Les séries Batraciens, Bestiaires ou Coquillages du potier Jean Girel (né en 1947) illustrent le talent de l'artiste pour imiter les peaux d'animaux. Son travail rend compte de la pertinence artistique d'une rencontre entre la chimie des matériaux de la céramique et le génie créatif de la vie organique. De même, Daniel de Montmollin (né en 1921) intègre à ses pâtes des cendres végétales pour inventer des émaux polychromes. Il envisage ainsi l'art de la poterie comme la confluence entre le minéral et le vivant, révélant, à l'échelle atomique, les processus d'une «biologie transfigurée ${ }^{13} \gg$.

Au XVIII ${ }^{\mathrm{e}}$ siècle, l'observation scientifique de la nature produit une foule d'images nouvelles, qui influence la représentation des animaux et des végétaux dans la céramique. Les illustrations naturalistes circulent et s'exportent des traités scientifiques aux décors de céramique, comme le montrent les services dits «de Buffon », produits à Sèvres entre 1782 et 1796 d'après les illustrations de François-Nicolas Martinet (1731-180?) réalisées pour l'Histoire naturelle des oiseaux de GeorgesLouis Leclerc de Buffon (1707-1788), publiée entre 1771 et 1786. L'achat par la Manufacture royale de Sèvres de ces planches en couleurs témoigne d'un changement d'attitude dans la représentation de l'animal en contexte décoratif : l'évocation fantaisiste laisse place à une recherche de fidélité aux modèles de la nature désormais plus facilement accessibles.

Vers 1731, le prince-électeur de Saxe Auguste le Fort (1670-1733) commande un large ensemble de sculptures animalières en porcelaine dure. L'attention portée par les sculpteurs Johann Gottlieb Kirchner (1706-1768) et Johann Joachim Kaendler (1706-1775) au réalisme des animaux, qu'ils étudient dans la ménagerie de Dresde, doit ici servir le prestige d'un prince cherchant par une commande monumentale à affirmer sa puissance et à promouvoir la finesse exceptionnelle de la porcelaine de Saxe nouvellement mise au point dans les ateliers de Meissen. Les ménageries, avant d'être des lieux d'étude des animaux, sont des parcs d'agrément, visant à la fois à montrer la richesse du prince et l'étendue de son pouvoir sur le reste du monde ${ }^{14}$. La forme de celle de Versailles rappelle d'ailleurs celle d'un panoptique. Établissant la domination symbolique de l'homme sur l'animal et du prince sur le reste du monde, la ménagerie montre que l'enjeu de la possession, de la vision et de la représentation des animaux est avant tout politique ${ }^{15}$.

En France, le Muséum d'histoire naturelle, fondé sous la Révolution, accueille les animaux transférés depuis la ménagerie de Versailles. Des artistes romantiques, au premier rang desquels Antoine-Louis Barye (1795-1875) et Eugène Delacroix (1798-1863), travaillent vers 1830 à une nouvelle forme de représentation de l'animal, fondée sur l'observation, le dessin des modèles vivants ou la prise de mesure sur des cadavres ${ }^{16}$. Chez Antoine-Louis Barye, cette recherche d'exactitude des proportions est néanmoins mise au service de compositions figurant des combats largement théâtralisés ${ }^{17}$. La sculpture animalière se diffuse progressivement, à partir de 1830, sous forme d'objets décoratifs en bronze et en porcelaine de petites dimensions, adaptés aux intérieurs bourgeois. À Limoges, le sculpteur Paul Comoléra (1818-1897) fournit aux manufactures de porcelaine de 
nombreux modèles d'oiseaux et de chiens tantôt employés comme décor d'une pièce de forme, tantôt présentés comme sculptures autonomes. Au début du $\mathrm{XX}^{\mathrm{e}}$ siècle, la céramique devient un outil de diffusion précieux de sculptures animalières plus accessibles, notamment les œuvres de Paul Jouve (1878-1973) et Édouard-Marcel Sandoz (1881-1971), qui continuent à travailler d'après des modèles animaux vivants. De l'animal romantique terrifiant qui incarnait une fascination idéalisée de la violence à l'état sauvage ${ }^{18}$, on passe à une image de l'animal stylisée, adoucie et plus conforme avec le confort et l'apaisement que le foyer moderne doit offrir. Parallèlement au développement des ménageries et des zoos, la présence croissante d'objets d'art animalier incarne sans doute un changement du rapport à l'animal qui s'opère en Occident à partir du début du XIX ${ }^{\mathrm{e}}$ siècle à mesure que l'industrialisation et l'urbanisation éloignent l'homme du contact avec l'animal, autrefois incontournable ${ }^{19}$.

\section{Imaginaire organique}

L'énergie qui se dégage des végétaux et des animaux, de leur croissance et de leur prolifération inspire un jaillissement d'inventions formelles qui s'affranchissent des modèles offerts par la nature. La copie d'une forme fait alors place à l'imitation d'un processus biologique qui nourrit la céramique, dont les techniques sont employées tour à tour dans une optique de stylisation, d'abstraction ou d'hybridation.

Au début du XVIII ${ }^{\mathrm{e}}$ siècle, des ornemanistes inventent le style rocaille, un langage décoratif qui détourne, contorsionne et exagère avec virtuosité les formes de la nature. Ce style donne naissance à des «caprices » qui restituent les formes surprenantes, complexes et insaisissables des coquillages, végétaux et minéraux. Pour les manufactures de céramique de l'époque, la porcelaine (dont le nom provient de la proximité visuelle et tactile avec le coquillage éponyme), et surtout la faïence fine, dont la plasticité rend possible le modelage de découpes complexes, constituent un terrain d'expression particulièrement adapté à ce goût rocaille qui caractérise les arts décoratifs français de la première moitié du XVIII ${ }^{\mathrm{e}}$ siècle. La saucière dite « Duplessis », du nom du créateur qui la dessina en 1756 pour la manufacture de Vincennes, est faite d'un mélange de corail, de feuillage et d'une vague sur le point de casser ${ }^{20}$. Cette invention formelle, réalisée probablement plus dans un but décoratif que scientifique, s'apparente néanmoins à l'intuition artistique d'un continuum reliant animal, végétal et matière inerte, perceptible dans de très nombreuses pièces de cette période.

A la fin du XIX ${ }^{\mathrm{e}}$ siècle, les protagonistes français et belges de l'Art nouveau, tels Hector Guimard (1867-1942) ou Victor Horta (1861-1947), se distancient du langage visuel ornemental de leur époque et remettent au goût du jour l'utilisation des formes végétales dans un esprit de stylisation et d'abstraction inédit. La profonde rénovation visuelle que constitue l'Art nouveau, qui envahit l'architecture, le mobilier urbain et les intérieurs publics et privés des grandes villes, puise son inspiration dans un nouveau regard porté sur la faune et la flore. À Sèvres comme à Limoges, la porcelaine se prête au jeu de la ligne en coup de fouet. Elle se charge d'une expressivité indissociable d'un intérêt pour les logiques organiques qui ordonnent et structurent le monde du vivant.

Le vase des Pommerets conçu par Anatole Fournier vers 1896 au sein de la manufacture nationale de Sèvres est issu de l'interprétation libre d'un bulbe végétal. La version proposée par le décorateur Henri Barbéris, marquée par un mouvement hélicoïdal, illustre «l'effacement de la dualité structuredécoratif en faveur d'une composition décorative naturaliste où se fondent forme et décor ${ }^{21}$. » Inspirée à l'origine à partir de lis et finalement réalisée avec des feuilles de chicorée, cette déclinaison témoigne d'un regard plus intéressé par l'évocation de la dynamique d'une floraison que par la restitution exacte d'une espèce en particulier.

À la fois artiste et botaniste, Émile Gallé (1846-1904) possède une connaissance scientifique de la biodiversité nancéienne. Son regard amoureux des insectes et des plantes le pousse à s'en inspirer pour 
trouver des formes et des textures nouvelles pour les objets précieux qu'il produit, en verre, en céramique ou en bois. Loin de la vision statique de la fleur épanouie souvent privilégiée dans les arts décoratifs, Émile Gallé s'intéresse au cycle complet du vivant de la naissance à la mort en passant par la croissance et la dégénérescence, et aux variations formelles que lui inspirent ces différents états ${ }^{22}$. Sa connaissance des méthodes et des outils de la science ne l'empêche pas de promouvoir une vision poétique de l'art à mille lieux de la seule recherche de fidélité avec le modèle observé : «Le document naturaliste le plus scrupuleux, reproduit dans un ouvrage scientifique ne nous émeut pas, parce que l'âme humaine en est absente ; tandis que la reproduction, cependant très naturelle de l'artiste japonais par exemple, sait traduire de façon unique le motif évocateur ou le minois, tantôt moqueur, tantôt mélancolique de l'être vivant, de la chose pensive ${ }^{23}$. »

À l'attention scientifique portée aux processus de la nature s'ajoute en effet l'influence des œuvres japonaises qui font sensation aux Expositions universelles à partir des années $1860^{24}$. La vivacité du trait et l'esprit de synthèse des estampes japonaises inspire des décors de céramique chez Émile Gallé et Félix Bracquemond. L'emploi du grès et le recours à des surfaces texturées ou à des formes biomorphiques nourrit le travail de céramistes comme Alexandre Bigot (1862-1927), Pierre-Adrien Dalpayrat (1844-1910) ou Ernest Bussière (1863-1913). Au-delà des considérations stylistiques, c'est un nouvel imaginaire du vivant plus proche de la nature qui est apprécié par les artistes occidentaux ${ }^{25}$.

L'intérêt plastique pour les phénomènes de croissance, de tension, de rétractation et de boursouflure d'un corps vivant est toujours présent chez de nombreux artistes contemporains tels que Claire Lindner, Johan Creten ou Farida Le Suavé. La céramique semble être un art particulièrement propice à faire advenir des formes qui évoquent le vivant sans pour autant s'y référer directement. Il s'agirait d'une «abstraction organique », où formes et textures font écho au vivant tout en évitant sa représentation littérale. En histoire de l'art, le terme «biomorphisme » renvoie en général à une tendance née dans les années 1930 à la croisée du surréalisme et de l'art abstrait, favorisant les formes arrondies, suggérant la vie par opposition à la géométrie orthonormée. Jean Arp (1886-1966), l'un des grands représentants de ce courant, a travaillé à la manufacture nationale de Sèvres entre 1963 et 1966 où il a conçu plusieurs formes de vases synthétisant l'idée d'un torse humain. La pertinence de cette alliance entre forme biomorphique et porcelaine est encore plus frappante chez Wayne Fischer, dont les sculptures renvoient selon les cas à la tension musculaire d'une articulation, à la sensualité d'une muqueuse. Pour pouvoir modeler et travailler en volume, l'artiste ajoute de la fibre à la porcelaine pour en accentuer la tenue. Ses pièces sont ensuite émaillées au pistolet puis rendues mates par sablage après cuisson, offrant ainsi à aux sculptures une douceur laiteuse ${ }^{26}$.

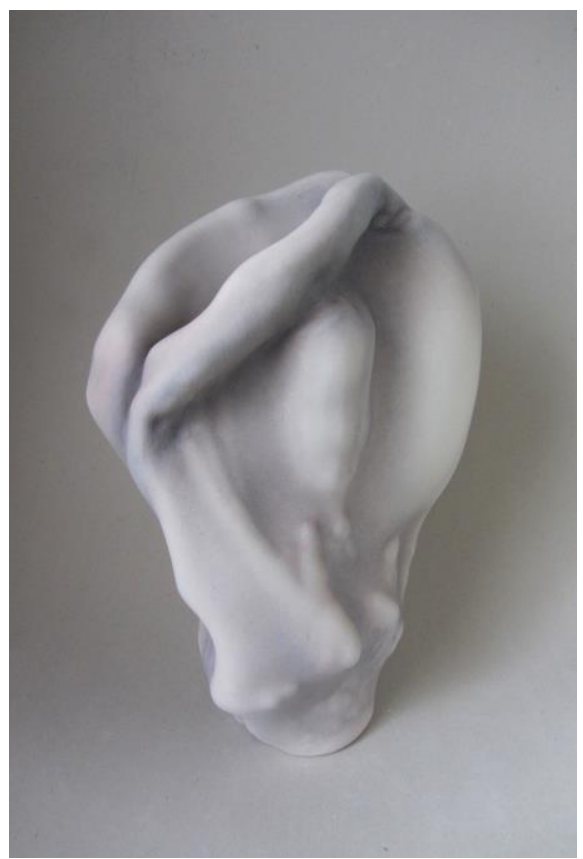


De la cuisson, moment d'une métamorphose décisive, surgissent bien souvent des déformations, des créatures étranges ou monstrueuses dont les artistes et les artisans travaillant la céramique ont su tirer profit pour inventer des formes nouvelles. Cherchant à ouvrir la sculpture à de nouvelles expériences de matière et de couleur, des artistes de la fin du XIX ${ }^{\mathfrak{e}}$ siècle tels que Jean Carriès (1855-1894) ou Jean-Désiré Ringel d'Illzach (1847-1916) travaillent le grès et les émaux pour inventer des textures épidermiques qu'ils appliquent à des animaux fantastiques. Dans un contexte artistique marqué par le symbolisme, le goût du monstrueux qui s'exprime chez certains céramistes fait étrangement écho aux bouleversements engendrés par la théorie de l'évolution de Charles Darwin (1809-1882). De la littérature fantastique au cinéma de science-fiction, l'imaginaire de la chimère connaît un succès croissant au cours du $\mathrm{XX}^{\mathrm{e}}$ siècle et ouvre des pistes de réflexion fructueuses pour la pensée du vivant, de ses origines et de son devenir. À l'image des créatures hybrides de Jean Fontaine, qui mêlent animal, empreintes de corps humain et éléments mécaniques, la céramique contemporaine peut être le lieu d'un regard critique porté sur la biodiversité et les manipulations du vivant (clonage, organismes génétiquement modifiés) qui agitent aujourd'hui la communauté scientifique et l'opinion publique.

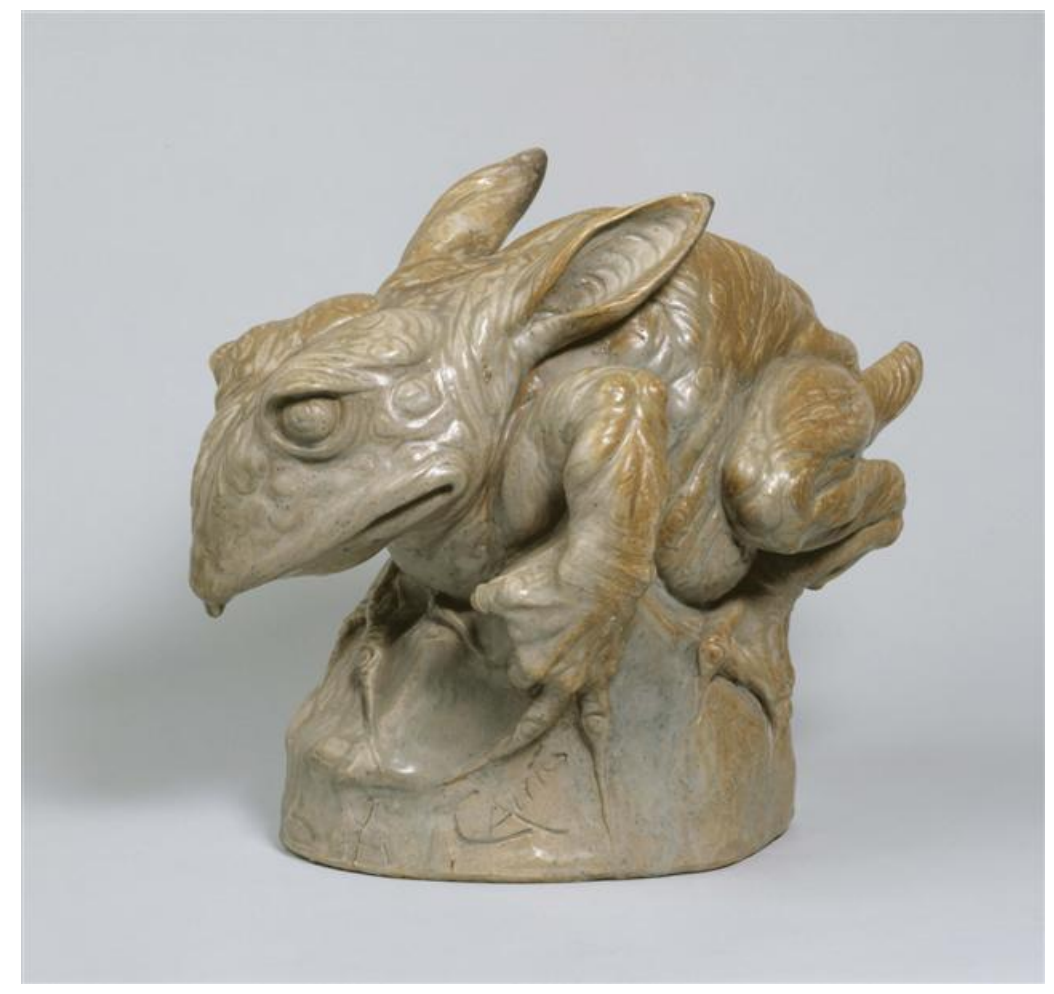

Jean Carriès, Grenouille à oreilles de lapin, grès, 1891, Petit Palais - Musée des Beaux-Arts de la ville de Paris, crédit photo : Patrick Pierrain/Petit Palis/Roger-Viollet.

\section{3. À l'intérieur du vivant}

La représentation des organes ou des mécanismes internes des êtres vivants est souvent plus confidentielle ou circonscrite à des travaux médicaux. Elle attise pourtant la curiosité des artistes qui s'approprient les formes invisibles et les processus cachés du vivant. Dans la céramique s'incarnent alors différentes réflexions esthétiques et éthiques sur la définition de la vie, ses limites ou la maitrise que peut en avoir l'homme.

La terre, lorsqu'elle est manipulée et modelée par les mains de l'artiste, s'apparente parfois directement à la chair. Cœur, os, réseaux sanguins ou musculature composent en céramique un paysage fragmenté du corps parfois proche de l'étude chirurgicale et de la dissection. Des artistes contemporains, comme Valérie Delarue ou Marc Alberghina, exploitent les nuances et l'aspect luisant 
de l'émail pour représenter des organes isolés. À la fois fragiles et monstrueux, ces cœurs et ces langues évoquent l'intimité de l'intérieur du corps, habituellement caché. En contexte scientifique, la pratique de la dissection, qui dévoile l'intérieur de l'animal ou du corps humain, procède d'une logique similaire. Les illustrations anatomiques sont parfois traitées comme des œuvres à part entière, dépassant leur objectif descriptif et pédagogique pour devenir des images autonomes. Les planches dessinées par Jacques-Fabien Gautier d'Agoty (1716-1785) pour la Myologie complète en couleur et grandeur naturelle (1746) illustrent autant un souci de composition artistique qu'une fascination esthétique pour l'intérieur du corps remarquée au XX $\mathrm{XX}^{\mathrm{e}}$ siècle par les surréalistes. De même, la bizarrerie des écorchés d'Honoré Fragonard (1732-1799), réalisés au moyen d'une technique d'injection vasculaire $^{27}$ nourrit l'imaginaire de l'artiste contemporain Michel Gouéry dont les sculptures en céramique donnent régulièrement à voir des viscères accumulés ou proliférants.

L'invention du microscope au XVII ${ }^{\mathrm{e}}$ siècle permet la découverte et l'observation d'êtres jamais vus auparavant. Ouvrage majeur de l'histoire de l'illustration naturaliste, Micrographia, publié en 1665 par le savant anglais Robert Hooke (1635-1703), devient rapidement un best-seller en raison du caractère spectaculaire de ses gravures. Le vivant invisible fait l'objet d'un changement d'échelle à la fois didactique et fantasmagorique. Plus tard, le biologiste allemand Ernst Haeckel (1834-1919) publie en 1902 Kunstformen der Natur, un ouvrage resté célèbre pour les planches révélant l'existence de planctons et d'êtres vivants unicellulaires dont les formes extraordinaires fascinent les artistes ${ }^{28}$. Les exosquelettes blancs des radiolaires en particulier inspirent au céramiste contemporain suisse Arnold Annen des formes organiques attirantes par leur perfection géométriques et hostiles par la transposition à l'échelle humaine d'excroissances pointues. La porcelaine remplace ici le dioxyde de silicium et rappelle l'utilisation créative du minéral par le vivant dans la fabrication d'architectures complexes. L'invention de formes proches de celles d'organismes unicellulaires, des bactéries ou des virus peut également résulter d'une intuition purement plastique : en 2011, l'exposition Céramiques vivantes, fruit de la collaboration entre un céramiste, Jean-Michel Barathon-Cadelle, et un enseignant chercheur en biologie de l'université Blaise Pascal de Clermont-Ferrand, Philippe Bouchard, expliquait certains mécanismes de morphogenèse (symétrie, importance de la sphère, exosquelette), et permettait de se familiariser avec le raku, une technique basée sur le refroidissement brutal de la céramique. L'univers artistique d'un sculpteur est ainsi entré en résonance avec des observations scientifiques.

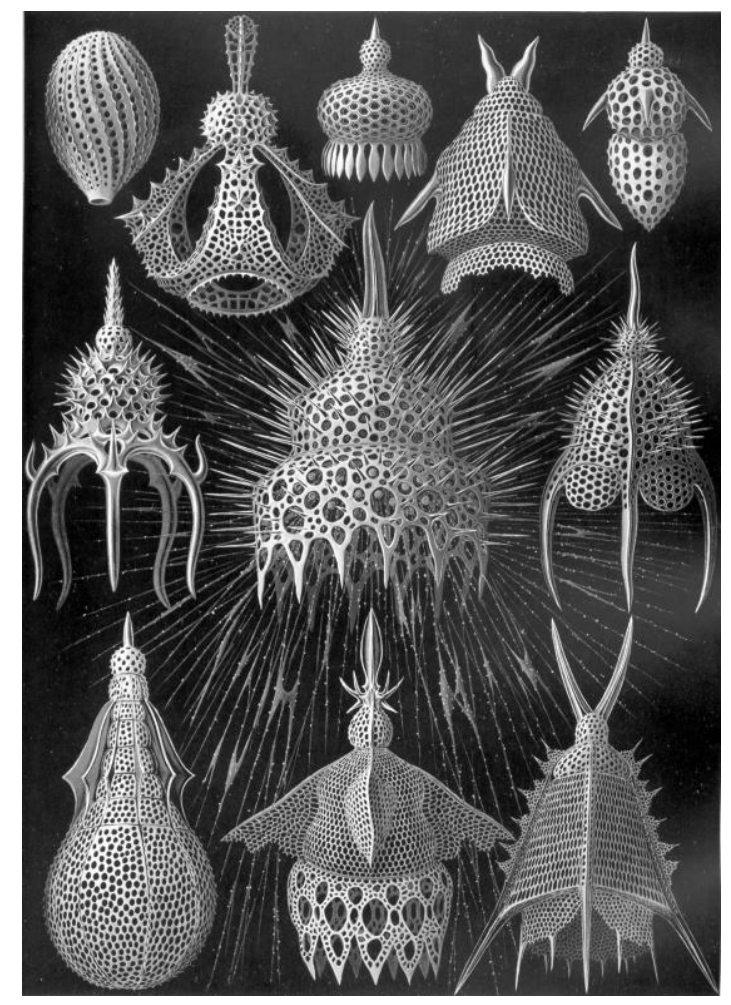


Des lois de Mendel aux « ciseaux moléculaires » CRISPR-Cas9 en passant par la forme en double hélice de l'ADN identifiée par Rosalind Franklin, James Watson et Francis Crick en 1953, la découverte progressive des lois de la génétique a permis d'envisager le vivant comme un «programme » sur lequel il est possible d'intervenir.

Parallèlement, la révolution numérique a offert de nouvelles perspectives d'imitation de la nature : à la croisée du design, de la médecine et du génie génétique, il est désormais possible d'imprimer en 3D des tissus vivants à partir de cellules-souches. Des formes scannées ou conçues informatiquement peuvent aussi être produites en céramique via l'utilisation d'imprimantes 3D adaptées à la pâte d'argile ou de porcelaine. Il est alors possible de bénéficier à la fois de la puissance de calcul de l'informatique et des propriétés physiques de la céramique pour créer des prothèses médicales biocompatibles. À Limoges, l'expertise technique héritée de plus de 250 ans de production porcelainière débouche depuis quelques années sur ces applications techniques innovantes. Une politique de transfert de technologies entre l'Institut de recherche sur les céramiques (IRCER) et la société 3DCeram a permis la mise au point, en 2005, d'implants crâniens en céramique imprimée par stéréolithographie laser, système inventé par le chercheur Thierry Chartier ${ }^{29}$.

L'emploi de la céramique en contexte biomédical permet d'imaginer comment on peut réparer le corps grâce à des éléments artificiels. On trouve ainsi toujours de nouvelles façons d'éditer, de réparer et de modifier les corps humains. Ce constat, qui peut légitimement être source d'inquiétude et ouvrir la voie à de nouvelles formes de l'exploitation des humains, montre aussi notre intégration à un environnement profondément technicisé.

Les codes et algorithmes permettant de simuler artificiellement la croissance du vivant et de mémoriser des formes complexes dématérialisées stimulent autant la recherche scientifique que la création artistique. Autour de 2010, une technique d'impression céramique dérivée de l'extrusion de fil chaud se développe, grâce notamment aux expérimentations du studio anversois $\operatorname{Unfold}^{30}$, qui recherche alors des passerelles entre artisanat et technologies numériques. Facilitée par Internet et par la publication de données en libre accès dans un esprit collaboratif, cette pratique se diffuse et donne naissance à des œuvres originales. Les céramiques imprimées en 3D du potier Jonathan Keep, dont certaines ont été acquises en 2017 par le Musée national Adrien Dubouché, sont le fruit d'une réflexion sur la forme et le vivant. Son installation Seed bed, littéralement « lit de graines », renvoie en effet à l'idée d'une germination, d'une croissance organique, ou d'une forme prête à éclore. Pourtant, loin d'avoir été copiées d'après nature, ces formes ont été inventées de toutes pièces par Jonathan Keep ${ }^{31}$, à l'aide de fonctions mathématiques, les harmoniques sphériques. Dans le champ de l'architecture expérimentale, l'architecte américaine Jenny Sabin développe depuis 2014 un projet baptisé Polybrick, mêlant céramique imprimée en 3D, biologie et recherche d'une architecture intelligente. Après avoir mis au point un modèle de brique inspiré de l'ostéogenèse, sa recherche évolue aujourd'hui vers l'intégration sur des tuiles en céramique d'un ADN de synthèse envisagé comme un porteur programmable d'information ${ }^{32}$. Ses travaux ouvrent des pistes qui conduisent non seulement à la création de formes complexes et originales, mais aussi à l'invention d'architectures vivantes, efficientes énergétiquement et capables de réagir à l'environnement. 


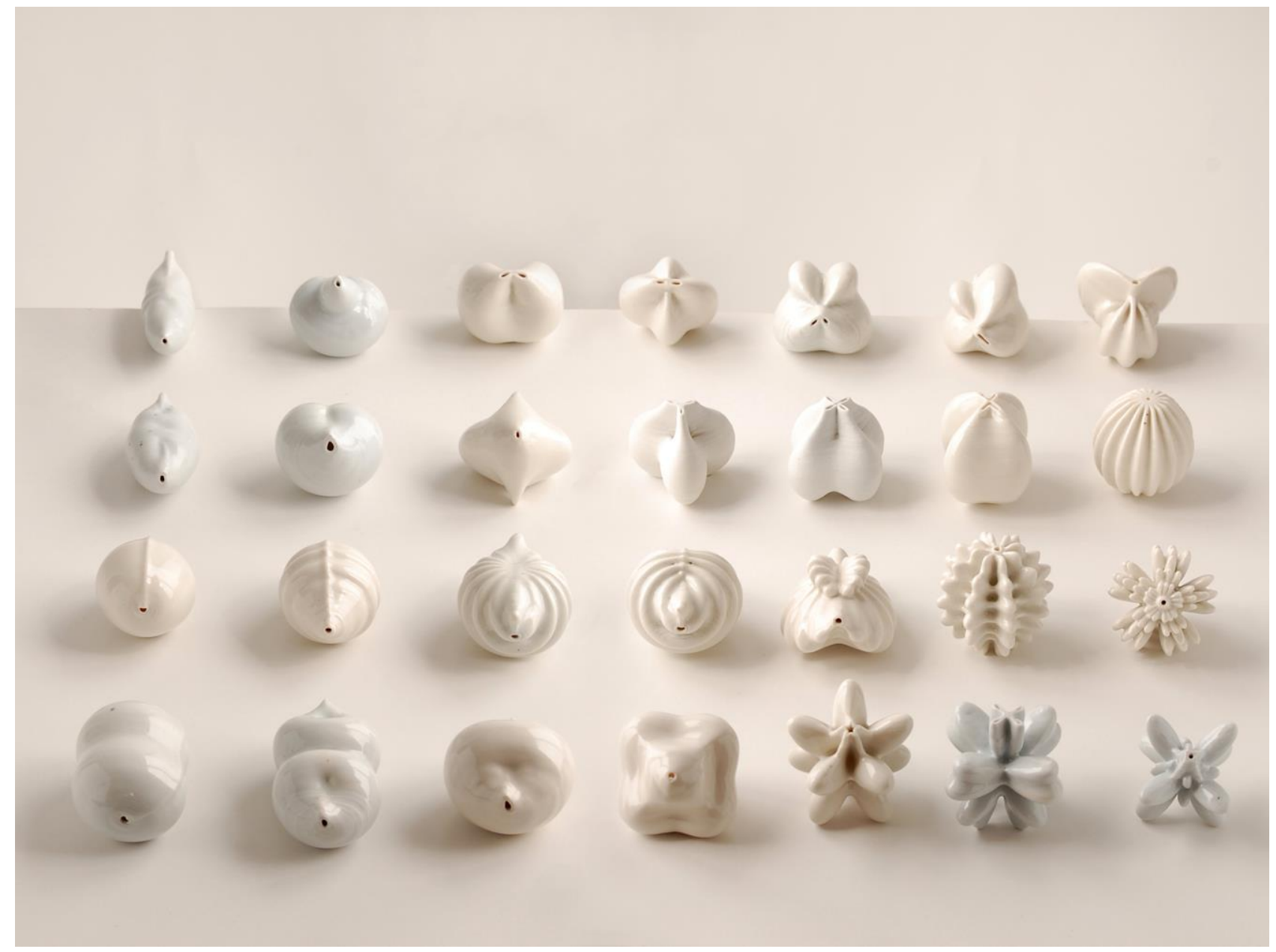

Jonathan Keep, Seed Bed, porcelaine imprimée en 3D, 2013, Musée national Adrien Dubouché, Limoges, crédit photo : Jonathan Keep.

\section{Conclusion}

La recherche de naturalisme permet donc en céramique de saisir le vivant, de s'en approcher et d'en fixer les formes pour le comprendre. Cette ambition révèle parfois également un rapport de captation voire de possession du vivant qui s'inscrit dans une histoire au sein duquel l'homme, son regard et sa soif de connaissances occupent une place centrale.

Le vivant est par ailleurs un territoire fertile pour l'imaginaire dont le caractère modelable s'accorde avec la plasticité de la céramique. L'exploration des métamorphoses organiques génère non seulement des formes nouvelles mais aussi des zones « inter-règne » qui invitent à repenser le vivant.

À la confluence de l'art de l'ingénierie et de la biologie, la céramique est également une porte d'entrée vers des processus cachés du vivant qu'elle rend visible. Sa pratique permet de nouer des alliances avec du vivant non-humain, voire avec du vivant artificiel.

Ces recherches invitent à considérer le vivant, à la suite de Donna Haraway, au sein d'une «nature » qui serait plutôt une «natureculture », c'est-à-dire non un lieu hors-temps, dépourvu d'histoire et radicalement autre, mais un environnement dans lequel les humains prennent place et auquel ils se relient de façons diverses au cours de l'histoire ${ }^{33}$.

De nombreux objets, comme les spécimens de sciences naturelles ou les animaux de compagnie ${ }^{34}$, ne sont ni «naturels » ni «culturels » : ils participent des deux, et peuvent être vus comme des sites de renégociation de la frontière entre nous, humains, et ce qu'il est convenu d'appeler «nature ». Cette 
renégociation est un enjeu majeur. L'art peut alors se manifester comme un discours «natureculturel » sensible. L'histoire de la céramique contribue ainsi à une histoire du rapport au vivant, nourrie de l'histoire de l'art et de l'histoire des sciences.

Ce qui peut être commun à des disciplines aussi éloignées que la biologie et l'histoire de la céramique nous permet de rapprocher ces domaines au service de savoirs qui les dépassent ${ }^{35}$.

Les dynamiques morphogénétiques, dont on a vu qu'elles étaient un sujet exploré par les artistes pratiquant la céramique, mettent en jeu à la fois la biologie et l'histoire de l'art. Les formes sont le résultat de contraintes, mais aussi d'une histoire. En s'intéressant aux études scientifiques sur la morphogenèse ${ }^{36}$ et aux écrits d'historiens $\mathrm{d}^{\prime} \operatorname{art}^{37}$ et de philosophes ${ }^{38}$, on voit émerger un champ transdisciplinaire de l'étude des formes.

Toutes ces formes vivantes nous amènent à un moment de l'histoire qui voit la notion de vivant être profondément remise en question, non seulement dans les sciences mais aussi dans l'imaginaire collectif.

Les procédés techniques et les possibilités d'inventions formelles propres à la céramique contribuent à troubler les catégories (espèces, branches de l'évolution, règnes) et les frontières (minéral, organique) du vivant. Ce constat fait écho aux recherches scientifiques récentes mettant en évidence toujours plus d'éléments (cristaux, virus, tissus vivants artificiels, etc.) dont les propriétés physiques ou le comportement déstabilisent les définitions qui structurent notre rapport au monde.

Cette remise en question nous fait nous intéresser de plus en plus à l'idée d'un continuum vivantnon-vivant, qui existait déjà, quoique sous une autre forme, chez Bernard Palissy. Privilégier l'idée de continuité plutôt que celle de catégorie invite à se concevoir comme partie intégrante de la matière du monde et de ses transformations. C'est à cette condition que nous pouvons entrevoir des rapports d'empathie avec les autres membres de la communauté du vivant.

L'intérêt du designer François Azambourg ${ }^{39}$ pour les termitières va dans le sens de cette réflexion. L'emploi de la terre est en effet caractéristique de l'activité des termites du Burkina Faso. Ces dernières, en digérant la terre, la filtrent et lui donnent une qualité spécifique, que les potiers locaux utilisent pour la céramique. L'idée d'Azambourg est de se servir de la partie émergée de la termitière, séchée par le soleil, qui conserve l'empreinte des galeries construites par les termites, comme matériau de création. Il met ainsi en évidence nos points communs avec ces animaux : le travail de la terre, mais aussi la nécessité d'habiter et la pratique de l'architecture qui en découle. Elle fait de la technique et de l'habitat non des spécificités culturelles humaines mais des besoins communs à tous les vivants.

\section{Notes}

\footnotetext{
1 Marie-Christine Maurel, Les origines de la vie, Le Pommier, Paris, 2017, p. 125.

2 Jean Girel, La sagesse du potier, JC Béhar, Paris, 2004, p. 18.

3 «La mémoire de la porcelaine est redoutable. C'est un phénomène bien connu à Sèvres, où les anses des tasses sont collées de travers, suivant un angle propre à chaque tourneur ; la pièce se dévissant proportionnellement au vissage qu'elle a subi, l'anse retrouve la verticale après cuisson », ibid., p. 48.

4 Voir Christophe Bonneuil «Le siècle du gène » in Histoire des sciences et des savoirs, sous la direction de Dominique Pestre, Seuil, Paris, 2015, pp. 297-317.
}

${ }^{5}$ La paternité de ce terme est attribuée à Ernst Haeckel, en 1866. 
${ }^{6}$ Ces recherches liant art et vivant font l'objet depuis quelques années de publications et d'expositions parmi lesquelles on compte notamment Bêtes Off (Paris, Musée de la conciergerie, 2011), Beauté animale (Paris, Grand Palais, 2012) pour la question de l'animal ; Jardins (Paris, Grand Palais 2017), Jardins infinis, (Paris, Centre Pompidou Metz, 2017) pour la question du végétal et du paysage ; ou bien En vie/Alive - Aux frontières du design (Fondation EDF 2013), Naturaliser l'architecture (Orléans, FRAC Centre, 2014) ; La Fabrique du vivant (Paris, Centre Pompidou, 2019) pour la question de la morphogenèse et du design bio-inspiré.

${ }^{7}$ Marie-Noëlle Bourguet, Pierre-Yves Lacour, «Les mondes naturalistes : Europe (1530-1802), Dominique Pestre dir., Histoire des sciences et des savoirs, Stéphane Van Damme dir., Tome I : De la Renaissance aux Lumières, Seuil, Paris, 2015, p. 256.

8 Rafael Mandressi, «Cultures visuelles des sciences », ibid., p. 238.

${ }^{9}$ Léonard N. Amico, À la recherche du paradis terrestre. Bernard Palissy et ses continuateurs, Flammarion, Paris, 1996, p. 42.

${ }^{10}$ Pour une analyse plus complète de la pensée théorique de la nature de Bernard Palissy, voir Léonard N. Amico, À la recherche du paradis terrestre. Bernard Palissy et ses continuateurs, Flammarion, Paris, 1996, pp. 44-43. Voir également Philippe Morel, Les grottes maniéristes en Italie au XVI siècle, Macula, Paris 1998, pp.35-36

${ }^{11}$ Léonard N. Amico et Danielle Oger, «De l'histoire naturelle et de la nature historiée » in Un bestiaire fantastique, Avisseau et la faïence de Tours (1840-1910), Réunion des musées nationaux, Paris, 2002, pp. 53-58

12 «La nature y est conçue comme le théâtre d'affrontement mortels permanents : sur toutes les pièces, on peut relever plusieurs scènes de prédation ou d'affût. [...] En ce sens, ces céramiques sont bien de leur époque, celle de Malthus et de Darwin. Le XIX ${ }^{\mathrm{e}}$ siècle, marqué par l'industrialisation forcenée et son cortège de misères, conçoit la vie comme une lutte à mort permanente, le fameux struggle for life. » Pierre Cabard, «Faune et flore », in Bestiaire fantastique, Avisseau et la faïence de Tours (1840-1910), op. cit. p. 47.

${ }^{13}$ «Si le feu ne conserve que les minéraux de la plante, si ce processus de stérilisante minéralisation semble nous éloigner de la vie, serait-ce divaguer de se dire que cette agitation, cette danse plutôt de particules d'autant plus intenses que la température monte, ce va-et-vient des atomes qui se séparent pour se regrouper en vertu de nouvelles affinités, que cette intense vibration est en somme assimilable à une nouvelle biologie faisant écho à la vie de la plante, non pas retrouvée mais transfigurée elle aussi ? Une biologie ne se reproduisant que dans la ferveur d'une incandescence » Daniel de Montmollin, Pierres habitées, Éditions de la revue de la céramique et du verre, Vendin-le-Vieil, 2009, p. 52.

${ }^{14}$ Samuel Wittwer, The Gallery of Meissen Animals, Hirmer, Munich, 2006, p. 60.

${ }^{15}$ John Berger, «Why Look at Animals?», 1977, About Looking, Pantheon Books, New York, 1980, p. 21.

${ }^{16}$ Emmanuelle Brugerolles éd., Antoine-Louis Barye, "Le Michel-Ange de la Ménagerie », Carnets d'études $N^{\circ} 28$, Éditions des Beaux-arts de Paris, Paris, 2014.

17 «Barye met en scène des combats qui ne peuvent se produire dans le cadre du zoo. Certes, le tigre et le gavial sont tous deux issus du sous-continent indien, en revanche, la probabilité qu'un lion terrasse un sanglier ou qu'un jaguar dévore un lièvre est nulle. Tout son art se résume à la lutte pour la vie : celle que les animaux se livrent dans leurs féroces combats, et celle qu'il mène lui-même pour qu'ils aient, dans le bronze, l'apparence la plus vivante possible. » Emmanuelle Héran, «Antoine-Louis Barye, Tigre dévorant un gavial» in Beauté Animale, Réunion des musées nationaux, Paris, 2012, p. 210.

18 « The treatment of animal in 19th century romantic painting was already an acknowledgement of their impending disappearance. The images are of animals receding into a wildness that existed only in the imagination. » John Berger, «Why Look at Animals? », op. cit., p. 17.

${ }^{19}$ John Berger, « Why Look at Animals? », op. cit., passim.

${ }^{20}$ Tamara Préaud et Antoine d'Albis, La Porcelaine de Vincennes, Paris, Adam Biro, 1991, notice diffusée sur le site du musée des Arts décoratifs de Paris : https://madparis.fr/francais/musees/musee-des-arts-decoratifs/parcours/xviie-xviiiesiecles/la-rocaille/sauciere-duplessis

${ }^{21}$ Isabelle Laurin, «L'Art nouveau à Sèvres, l'école Guérin et le vase des Pommerets », Revue de la société des amis du musée national de céramique, $\mathrm{N}^{\circ} 19,2010$, p. 132-142.

${ }^{22}$ Valérie Thomas, «Emile Gallé, de la nature à l'atelier », Organismi, cat. exp. Galerie d'Art moderne de Turin, Skira, Milan, 2016.

${ }^{23}$ Emile Gallé, discours du 17 mai 1900 à l'Académie Stanislas de Nancy, cité par Henri Claude, « le décor symbolique », dans Emile Gallé, nature \& symbolisme « influence du japon », catalogue de l'exposition Vic-sur-Seille, 2009, p. 25. 
${ }^{24}$ Lionel Lambourne, Japonisme, échanges culturels entre le Japon et l'Occident. Phaidon, Paris 2006, pp. 68-83.

${ }^{25}$ «C'est donc au moment précis où la culture occidentale cherchait à établir un rapport de type nouveau avec son milieu naturel qu'elle prit connaissance de la façon dont les japonais l'appréhendaient dans leur art. [...] Les japonais d'avant la modernisation du pays ne distinguent pas, eux, de façon aussi précise entre éléments naturels d'un côté et éléments purement humain de l'autre. » Akiko Mabuchi, «Japonisme et naturalisme » in Le Japonisme, cat. exp. Galeries nationales du grand Palais, 17 mai-15 août 1988, RMN, Paris, 1988, p. 34.

${ }^{26}$ Nicole Crestou, site Internet de l'artiste, https://mail.waynefischer.net/index.php/fr/

${ }^{27}$ Michel Lemire, «Fortunes et infortunes de l'anatomie et des préparatins anatomiques, naturelles et artificielles », in L'âme au corps, Réunion des musées nationaux, éditions Gallimard/Electa, Paris, 1993, pp. 70-98.

${ }^{28}$ Erika Krause, «L'influence de Ernst Haeckel sur l'Art nouveau » in L'âme au corps, op.cit., pp. 342-350.

${ }^{29}$ Voir la vidéo de présentation réalisée par le CNRS : https://www.youtube.com/watch?v=j35gAH2arRg

${ }^{30}$ Tamar Shafrir, «Unfold : Reinventing the Wheel », Michel Paysant dir., Les Arts du feu à l'heure actuelle, rencontres entre la céramique, le verre et les langages numériques, La céramique comme expérience, vol. 2, journées d'étude, ENSA, Limoges, 2017, Naima, publication numérique, pp. 53-79, https://www.naimaunlimited.com/biblio/la-ceramiquecomme-experience-vol-2/.

${ }^{31}$ Voir le site internet de l'artiste, particulièrement riche en documentation sur sa démarche et sa technique : http://www.keep-art.co.uk/index.htm.

${ }^{32}$ David Rosenwasser, Shogo Hamada, Dan Luo, Jenny E. Sabin, «PolyBrick 3.0: live signatures through DNA hydrogels », International Journal of Rapid Manufacturing, Vol. 7, Nos. 2/3, 2018, pp. 205-218.

${ }^{33}$ Delphine Gardey, «Donna Haraway : poétique et politique du vivant », Cahiers du Genre, N55, 2013, L’Harmattan, Paris, pp. 171-194.

${ }^{34}$ Ces deux exemples ont été étudiés par Donna Haraway, la taxidermie dans « Teddy Bear Patriarchy: Taxidermy in the Garden of Eden, New York City, 1908-1936 », Social Text, N 11, hiver 1984-1985, pp. 20-64, et la notion d'espèce de compagnie dans The Companion Species Manifesto: Dogs, People and Significant Otherness, Prickly Paradigm Press, Chicago, 2003.

${ }^{35}$ Voir la définition de la transdisciplinarité proposée par Francine Pellaud, professeur à la Haute école de pédagogie de Fribourg : https://www.canal-u.tv/video/canal_uved/4_multi_pluri_inter_ou_transdisciplinarite.19763

${ }^{36}$ Paul Bourgine, Annick Lesne dir., Morphogenèse. L'origine des formes, Belin, Paris, 2006.

${ }^{37}$ Par exemple des historiens d'art issus de la tradition formaliste, tels qu'Henri Focillon, Vie des formes, PUF, Paris, 1943.

${ }^{38}$ On peut notamment citer Gilbert Simondon, L'individuation à la lumière des notions de forme et d'information, Millon, Grenoble, 2005 (publication de la thèse de Simondon, soutenue en 1958 et publiée en deux fois en 1964 et 1989). Cité par Sarah Margairaz, "Entre apeiron présocratique et métastabilité thermodynamique : l'idée de préindividuel chez Gilbert Simondon », Methodos. Savoirs et textes, № 13, 2013, en ligne, https://journals.openedition.org/methodos/3191\#ftn1.

${ }^{39} \mathrm{~L}$ 'artiste est actuellement en résidence à la Manufacture nationale de Sèvres, où il mène une recherche sur les termitières, dans le prolongement de la fabrication du trône en termitière réalisé en 2010 pour l'éditeur Poltrona Frau au festival parisien de design D’Days. 